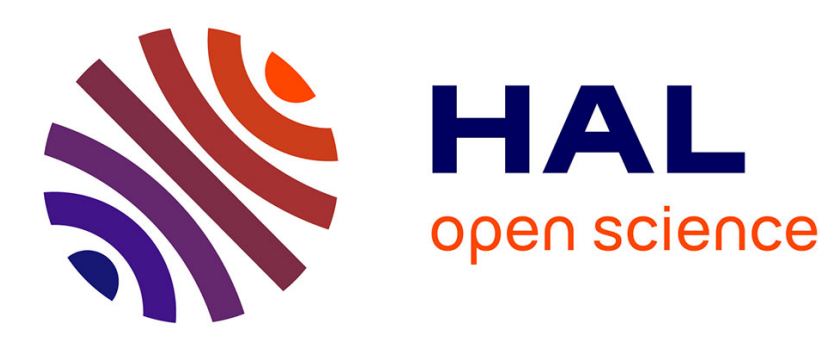

\title{
Don, autorité et reconnaissance dans la sociologie de l'évergétisme de Paul Veyne
}

\author{
Alice Le Goff
}

\section{To cite this version:}

Alice Le Goff. Don, autorité et reconnaissance dans la sociologie de l'évergétisme de Paul Veyne. Presses du Septentrion. Donner, reconnaître, dominer: trois modèles en philosophie sociale, pp.169172, 2016. hal-01418240

\author{
HAL Id: hal-01418240 \\ https://hal.science/hal-01418240
}

Submitted on 13 Jun 2018

HAL is a multi-disciplinary open access archive for the deposit and dissemination of scientific research documents, whether they are published or not. The documents may come from teaching and research institutions in France or abroad, or from public or private research centers.
L'archive ouverte pluridisciplinaire HAL, est destinée au dépôt et à la diffusion de documents scientifiques de niveau recherche, publiés ou non, émanant des établissements d'enseignement et de recherche français ou étrangers, des laboratoires publics ou privés. 


\section{Alice Le Goff (MCF Cerlis - Université Paris Descartes, Sorbonne Paris Cité) - Titre : «Don, autorité et reconnaissance dans la sociologie de l'évergétisme de Paul Veyne » - Version pre-print}

Dire que l'Essai sur le don de Marcel Mauss est un texte surchargé d'enjeux tant pratiques que théoriques relève de l'euphémisme. Dans ce texte, Mauss a mis l'accent tant sur l'importance - voire la centralité - que sur la complexité du don comme «phénomène social total »: si le détour par des sociétés «traditionnelles »s'impose pour mieux décrire les formes de l'échange non marchand que la notion de don recouvre, il n'en demeure pas moins que le don travaille jusqu'aux sociétés les plus industrialisées. Mauss y voit ainsi un « roc » de la société : à travers le cycle don/contre-don, il deviendrait possible de saisir ce moment fugace où «la société prend ». Mais de ce don, il s'est aussi agi de faire ressortir la dimension énigmatique et ambivalente. Enigmatique car comment comprendre cette obligation singulière, non seulement de donner et de recevoir mais de rendre, alors même que le don échappe en principe à la logique d'un échange commercial ? Ambivalente car le phénomène du don articule de façon étroite la spontanéité et l'obligation au point qu'il semble impossible de les dissocier. Bien plus, Mauss met aussi l'accent sur des ambivalences qui tiennent à la façon dont le don peut apparaître aussi bien comme un vecteur de production du lien social que comme un opérateur de stratification. Philosophes et chercheurs en sciences sociales n'ont pas cessé depuis de tourner autour de cette énigme et de ces ambivalences.

De fait, l'Essai entreprend notamment de mettre en lien plusieurs formes de don, en particulier le potlatch auparavant étudié par F. Boas et la kula mélanésienne dont Malinowski avait proposé une analyse. Le potlatch, observé entre autres dans le monde amérindien, désigne une fête qui rassemble soit toute une tribu, soit plusieurs tribus et donne lieu à des échanges de cadeaux sur un mode de rivalité agonistique pouvant aller jusqu'à la destruction de richesses. Mauss élabore à ce sujet la notion de «prestations totales de type agonistique » dont l'enjeu est la rivalité entre les chefs, la lutte pour l'honneur et le prestige ; ne pas rendre un potlatch revient à perdre la face et son statut de chef. Par contraste, la kula constitue une forme plus pacifique d'échange cérémoniel, de transactions rituelles structurées autour d'objets prestigieux mais sans valeur marchande (des colliers et des coquillages) et organisées en cercles. La kula exemplifie une figure du don différente de celle, placée sous le signe de la rivalité, à laquelle le potlatch est associé : elle correspond davantage au don comme alliance. Dans tous les cas néanmoins, on retrouve l'insistance sur la complexité du don comme processus impliquant différentes phases et obligations et sur l'articulation forte entre spontanéité et contrainte. Dans tous les cas aussi, les logiques de prestige sont au cœur des échanges capturés par les notions de potlatch et de kula.

Le contraste entre ces différentes formes de don explique en partie que le texte de Mauss ait pu faire l'objet de lectures si divergentes qui ont cherché à clarifier ce qu'est le don en le distinguant, mieux que Mauss lui-même ne l'avait fait, de ce qui n'est pas lui. Il s'est agi d'une part, d'aller plus loin dans la résolution de l'énigme du don, nombre de commentateurs s'accordant à souligner que l'interprétation maussienne du hau maori, comme force du donateur ou esprit accompagnant la chose donnée et obligeant à faire retour, est inaboutie et problématique. D'autre part, l'enjeu a été de mieux circonscrire le phénomène du don luimême. Cet effort est souvent allé de pair avec un durcissement de l'analyse, une tendance à gommer quelque peu les ambivalences du don en privilégiant certaines de ces facettes au détriment d'autres. Se sont ainsi affrontées des lectures axées sur les facettes plus agonistiques et hiérarchisantes du don et des lectures centrées sur la façon dont il produit des formes positives de réciprocité : les discussions autour du texte de Mauss ont été marquées, entre autres, par l'oscillation entre la dette vecteur de domination et la logique de 
mutualisation dont le don peut être un noyau et qui pourrait se faire le vecteur de pouvoir collectif.

Les travaux de Marcel Hénaff (2002 et 2012) illustrent la stratégie théorique consistant à privilégier le pôle de l'alliance : par contraste avec le don gracieux (unilatéral) et le don de solidarité, le don cérémoniel que Hénaff privilégie est caractérisé par sa dimension réciproque. Hénaff souligne sa radicale autonomie par rapport à l'échange marchand et la façon dont le don cérémoniel constitue une procédure de reconnaissance réciproque (articulant l'identification et le fait d'honorer autrui, de lui marquer de la considération); le don cérémoniel déjoue ainsi toute opposition entre société traditionnelle et société moderne et perdure au sein de nos sociétés industrialisées en tant qu'il fonde tout rapport à l'altérité ; il ne recouvre pas un échange de biens mais consiste dans le fait de se reconnaître publiquement comme partenaires d'échange.

A cette approche qui privilégie l'articulation entre don et reconnaissance comme alliance, s'opposent des perspectives plus centrées sur l'articulation entre le don et des formes plus agonistiques de reconnaissance. Claude Lefort (1951), par exemple, a proposé une interprétation du don comme lutte des consciences, comme acte libre d'un sujet à la conquête de sa propre subjectivité et assumant la prise de risque liée à la confrontation à autrui. Une telle lecture, en polémique avec Claude Lévi-Strauss et le structuralisme - aux yeux duquel le don est une nécessité dérivée de structures inconscientes de l'esprit humain - gomme les normes et contraintes enserrant les pratiques de don et contre-don. Ce n'est pas le cas de la lecture de Pierre Bourdieu (1972 et 1997) qui souligne la dimension contraignante du don mais s'écarte aussi de la réification structuraliste de l'échange de dons à laquelle il est reproché d'avoir gommé la temporalité du processus. Bourdieu rapproche don et dette soulignant le lien entre don et dépendance du donateur devenu obligé du donataire. Le don n'a rien d'un échange donnant-donnant au sens où rendre tout de suite quelque chose peut être interprété comme un signe de mépris. Don et contre-don sont nécessairement séparés par un laps de temps et c'est ce dernier qui permet de voiler les intérêts présidant à l'échange, de faire violence au donataire tout en masquant cette violence sous un masque de générosité. Le don est ainsi interprété comme vecteur de domination et comme l'un des opérateurs centraux de la conversion du capital économique en capital symbolique - suivant le vocabulaire de la sociologie bourdieusienne : le don est au cœur de toute opération de légitimation du pouvoir en ce qu'il redouble la domination économique par la sujétion morale. De Lefort à Bourdieu, le don est donc articulé à une forme agonistique et dissymétrique de reconnaissance plus qu'à une reconnaissance comme alliance (caractérisée par une forme potentiellement plus positive et symétrique de reconnaissance comme c'est le cas chez Hénaff).

Il n'est pas question ici de mettre en question l'apport de ces différentes lectures. Chacune, à sa manière, éclaire des logiques possibles du don. Sur le plan intellectuel, l'opération consistant à privilégier tel ou tel pôle du processus du don pour en explorer tous les aspects est d'ailleurs intellectuellement légitime et susceptible d'avoir des effets d'intelligibilité. Mais elle court aussi le risque de conduire à de fausses oppositions (entre le don comme reconnaissance et le don comme domination, entre le don comme reconnaissance-alliance et le don comme reconnaissance agonistique...) et d'évacuer la riche intuition des ambivalences du phénomène du don qui a caractérisé les analyses maussiennes.

Si j'ai choisi de revenir ici sur le travail de Paul Veyne dans Le pain et le cirque, c'est précisément que la sociologie historique de l'évergétisme qu'il y propose reste fidèle à cette mise en exergue des ambivalences des logiques du don ainsi que de la façon dont elles articulent reconnaissance et domination. A dire vrai, elles vont même encore plus loin dans la mise en exergue de ces ambivalences. Du fait d'une attention à la pluralité et à la variabilité historique des systèmes de don, elles ont également l'intérêt de court-circuiter le regard souvent trop normatif qui caractérise aussi bien les discussions relatives au «paradigme du 
don » ou aux intersections entre théorie néo-hégélienne de la reconnaissance et anthropologie du don. Sur la base d'une approche naviguant entre sociologie historique et anthropologie, Veyne a proposé une analyse d'un phénomène à la fois marginal et capital, montrant qu'il constitue un bon point d'entrée dans l'analyse de la structuration des comportements politiques : il s'agit de la pratique de l'évergétisme qui occupe une place très grande de 300 avant notre ère à 300 après et qui consiste dans le fait de donner à la collectivité des jeux, des monuments, du pain... Veyne distingue les différentes déclinaisons de cette forme civique de don de l'époque hellénistique à l'époque romaine et de la république romaine à l'empire. Il en propose une analyse historique attentive aux variations de l'évergétisme. Mais cette analyse est aussi le point d'appui d'une réflexion plus anthropologique et d'un travail de théorie sociale si l'on entend par là le dégagement de mécanismes sociaux susceptibles de nous en apprendre plus sur la structuration des comportements collectifs. Ce n'est pas un hasard si Jon Elster, lui-même adepte d'un tel type de démarche (et formé tout comme Veyne, par Raymond Aron), a puisé chez Veyne de riches intuitions à approfondir. C'est sous cet angle que le travail de Veyne m'intéresse: n'étant pas historienne, je n'aborderai pas ici les analyses de Veyne sous l'angle de leur apport à l'histoire de l'antiquité. C'est Veyne faisant œuvre de théorie sociale de manière fine, subtile et parfois sceptique qui fait ici l'objet de mon attention. Plus précisément, il s'agit de cerner son apport au questionnement, au cœur de cet ouvrage collectif, sur les modalités d'articulation possible entre don, reconnaissance et domination. L'analyse de l'évergétisme est traversée mezzo voce par la question de la reconnaissance au sens où, Veyne y revient sans cesse, la politique recouvre des rapports de domination qui sont aussi des rapports d'autorité et donc des rapports entre consciences. Et l'une des intuitions qui structurent le travail de Veyne sur l'évergétisme tient à l'idée suivante: le don n'est pas l'outil d'une production de la domination mais son effet, sa conséquence; le don est moins vecteur qu'effet et surtout expression d'une forme de domination. C'est cette intuition - qui, mine de rien, questionne une manière assez habituelle d'envisager le rapport entre don et domination - que j'explore dans ce texte : elle invite à interroger la combinaison entre conception instrumentale du don et vision manipulatoire des rapports de domination qui constitue un écueil tentant de la réflexion sur le sujet. Je commencerai par rappeler succinctement les grandes lignes de l'analyse de Veyne avant d'en faire ressortir les enjeux et implications.

\section{Evergétisme et pluralité des systèmes de don}

Rappeler les grandes lignes de l'analyse de l'évergétisme par Veyne, cela revient à insister sur la façon dont il distingue trois grandes configurations de l'évergétisme. L'évergétisme recouvre différents systèmes de don même si dans tous les cas, on retrouve l'importance de pratiques structurées autour du don de spectacles, de plaisirs publics, d'édifices. Veyne commence par reprendre à Mauss la notion de fait social total pour qualifier l'évergétisme et expliquer sa fascination pour ce phénomène qui peut relever d'une coutume autant que d'un point de droit, d'une attitude autant que d'un phénomène de mentalité et qui a des dimensions politique, sociale, dynastique, économique, religieuses, culturelles qu'il est malaisé de démêler. Veyne (1976: 26) tente d'emblée une définition formelle du phénomène : «on appellera évergétisme ou mécénat un système de contributions qui sont versées, spontanément ou du moins sans obligation formelle, par des personnes qui ont un intérêt quelconque, matériel ou spirituel, à la poursuite de l'objectif que ces contributions permettent d'atteindre ». L'évergétisme consiste en une pratique de don portant sur des biens collectifs. Cette pratique ne vise pas des individus, des groupes, une partie de la population (par exemple les pauvres) mais la collectivité dans son ensemble : un évergète offrira un banquet non pas à sa «clientèle » mais à la cité toute entière. L'évergétisme pourra être libre ou obligatoire car 
attachée à une fonction : on parle alors d'évergésie ab honorem. Mais, c'est un point sur lequel je reviendrai, Veyne insiste d'emblée sur la difficulté à objectiver la frontière entre la spontanéité et la contrainte : une évergésie en apparence libre peut être l'effet d'une " douce violence » et des mécanismes de conversion peuvent également advenir lorsqu'un notable par exemple donne parce qu'il devait donner mais donne plus qu'il ne devait donner. Dès le début de son livre, Veyne insiste sur les façons contradictoires dont l'évergétisme peut être envisagé que l'on y voit l'expression d'une lutte de distinction entre notables ou des formes de concessions des plus riches et puissants face à la pression de la plèbe. Aucune de ces représentations n'est totalement vraie, aucune n'est pour autant fausse, chacune a sa part de vérité : l'évergétisme articule des logiques différentes voire en tension les unes avec les autres; il constitue un phénomène ambivalent et Veyne ne recule jamais devant cette ambivalence. En découle une analyse qui dégage des «thèmes » permanents et leurs variations d'une période à une autre.

L'évergétisme des oligarques romains n'est pas celui des notables grecs, il obéit également à des logiques singulières de celles qui se dégagent de l'étude de l'évergétisme impérial. En effet l'évergétisme grec est un évergétisme de notables. Veyne s'inspire ici de la définition wébérienne du notable comme personne qui, de par sa situation économique, peut diriger la cité sans réclamer un salaire, qui ne vit pas de la politique mais pour la politique. Les cités héllénistiques sont des collectivités de petite taille, leur gestion n'implique pas de tâches trop techniques, ce qui rend possible un régime de notables auquel aboutit toute forme directe de démocratie. Dans ce cadre, le pouvoir des notables n'est pas « fonctionnel » et s'étend à toute la vie sociale. Veyne souligne qu'on est face à une forme sociale où le cumul des excellences et des pouvoirs va de soi : les échelles de prestige sont indistinctes, elles ne sont pas plurielles (contrairement à celles des sociétés capitalistes et «démocratiques ») de sorte que l'autorité des notables, enracinée dans leur richesse, s'étend à l'ensemble des secteurs de la vie sociale (1976: 127). Le notable est un chef «naturel» qui tient son autorité non pas d'une délégation, de l'hérédité ou de qualités personnelles singulières mais d'une puissance objective (en l'occurrence la richesse). L'activité politique représente pour lui une dignité cruciale: elle est au cœur de l'échelle de l'estime sociale, la richesse implique automatiquement le prestige et le pouvoir dans d'autres domaines et notamment dans le domaine politique. Les notables forment initialement une classe qui au fil du temps se transforme en ordre. Veyne revient sur le passage de l'oligarchie à un régime de notables. Ce passage est aussi celui du système liturgique à l'évergétisme. Les liturgies sont un mode de financement: elles peuvent être civiles et liées aux dépenses relatives notamment à des festivités religieuses; elles concernent alors la gestion et l'entretien d'un gymnase, d'un théâtre et d'un chœur, le financement de banquets ou de rituels divers. Elles peuvent aussi recouvrir des dépenses militaires ou de guerre. Les liturgies ne constituent pas un impôt au sens de contribution obligatoire sur le plan formel. Veyne récuse à ce sujet la distinction faite par Benjamin Constant entre liberté des Anciens et liberté des Modernes : non, le citoyen antique ne doit pas tout à la cité et une contribution fiscale systématique et obligatoire serait impensable. En principe le système repose sur le volontariat : les magistrats désignent les liturges parmi des volontaires. Et la liturgie est une forme d'honneur. Cependant le système repose bien sur une forme d'obligation informelle - il y a des seuils de fortune au-delà desquels il est difficile de se soustraire - et sa dimension contraignante s'est affirmée au fil du temps. A l'époque hellénistico-romaine, l'évergétisme prolonge sous certains angles le système liturgique mais s'en démarque en ce que cette dimension contraignante s'estompe (la Cité ne désigne pas des évergètes comme elle désignait les liturges même si l'accès à certaines magistratures implique par principe d'assumer les dépenses liées à l'exercice de la fonction). L'évergétisme a des ressorts communs avec la liturgie : il s'agit de dépenser pour être honoré mais cette logique honorifique est accentuée dans le cas de l'évergétisme du fait 
de l'assouplissement de la contrainte et de l'importance des honneurs officiels rendus aux évergètes (sous forme d'épitaphes ou de statues - que les notables honorés contribuent euxmêmes à financer). Les évergésies peuvent obéir à différents motifs : celui de la piété (on consacre aux dieux des monuments, des prix, des fondations); donner à la cité pour être honoré ; le motif du patriotisme qui conduit à financer les troupes, des campagnes militaires ; celui de la largesse $a b$ honorem amenant un magistrat à prodiguer des largesses à l'occasion de son entrée en fonction ou à assumer sur sa fortune personnelle les dépenses liées à sa fonction. Veyne souligne également l'importance des «pollicitations» ou promesses d'évergésie à visée électorale. Les charges publiques sont envisagées comme des honneurs pour lesquels on doit à la Cité des remerciements. Il n'est pas possible ici d'évacuer l'ambivalence des évergésies ab honorem qui recouvrent à la fois la nécessité de donner des gages de désintéressement à la collectivité et celle de payer pour faire fonctionner une « entreprise » appartenant aux détenteurs de fonctions. L'évergésie n'est pas un coût à payer pour accéder au pouvoir : on paie parce que l'on possède le pouvoir. Les fonctions publiques sont à la fois la propriété des notables, « leurs choses » et un honneur : elles le sont d'autant plus qu'elles sont réservées à une élite et n'impliquent des responsabilités qu'à une toute petite échelle, municipale. Veyne parle de «snobisme des dignités municipales »: "plus la politique devient médiocre, plus l'évergète devient un homme qui fait du bien à la cité essentiellement en ouvrant sa bourse » (1976: 264). A un certain degré, la fonction peut devenir un prétexte pour faire payer de telle sorte que gouverner égale donner. Plus la fonction devient un honneur «creux», plus elle appelle un paiement «secondaire» ou pourboire que Veyne nomme «contre-affect symbolique»: on retrouve l'idée que l'évergétisme est moins le prix que la conséquence des fonctions et honneurs publics. Il ne s'agit pas d'acheter le renoncement du peuple au pouvoir mais, au plus, de le rassurer sur le désintéressement de ceux qui le guident. Alors certes, les évergésies se présentent comme un hommage «qui monte » vers la cité, suivant un style civique et déférent de don allant du bas vers le haut mais leur motif fondamental est l'attachement à des fonctions qui sont autant de distinctions sociales coûteuses : «devenus maîtres exclusifs des cités, les notables, comme tous les privilégiés, se font un devoir et une doctrine de leur distance sociale » $(1976: 124)$ et l'évergétisme est pour les notables le mode d'expression de leur supériorité sociale. Il ne s'explique pas de façon psychologisante par la quête des honneurs : l'honneur est une pièce dans un système de distance sociale où l'évergésie ne crée pas la distance mais en découle (1976 : 280).

L'évergétisme des oligarques, à l'époque de la République, a des traits communs avec l'évergétisme grec mais prend une tournure différente. Les sénateurs romains ne sont pas des notables mais assument une «vraie» fonction politique pour laquelle ils ressentent de la fierté. Ils ne cultivent pas la distance sociale et se soucient peu de recevoir des statues pour avoir donné des jeux : ils veulent triompher, accéder à la fonction de consul, bref ils vivent de «grande politique ». Leur point d'honneur renvoie à l'accès à des fonctions de plus en plus élevées : il faut bien parler ici de dignitas mais pas de la dignité «bourgeoise » du notable ; la dignitas c'est la gloire, elle est proche de l'honneur médiéval si ce n'est qu'elle ne s'hérite pas mais se conquiert voire se démultiplie même au fil de la carrière politique. Les magistratures amplifient la dignitas et la res publica elle-même est aimée en tant que système qui la garantit. D'un côté, Veyne souligne bien que l'évergétisme ne peut se déployer au sens plein dans un tel contexte car il suppose que les fonctions publiques sont honorifiques. De l'autre, il admet qu'on est bien là face à une déclinaison spécifique de l'évergétisme comme moyen de rehausser la dignitas ou plus exactement mode d'extériorisation de la splendeur politique : par contraste avec l'évergétisme grec, la logique de l'évergétisme romain est plus politique que sociale. L'écart entre sénateur et plébéien est plus grand qu'entre les notables grecs et le peuple : les sénateurs romains n'ont pas besoin de prodiguer des largesses pour rassurer la 
plèbe, de donner des gages de désintéressement. Ils n'ont pas besoin d'être aimés par la plèbe mais désirent l'être. Il ne s'agit pas, via les évergésies, de plaire à de futurs électeurs mais d'acquérir du prestige "dans leurs têtes » et ce même s'ils sont relativement impuissants. Les évergésies semblent obéir à un désir de régner dans les consciences et pas seulement d'être obéi. Veyne distingue ici deux systèmes de don : les dons (de services et de bienfaits) internes à l'oligarchie et les dons au style condescendant entre l'oligarchie et la plèbe. Veyne insiste sur les cadeaux symboliques aux soldats, sur les évergésies des triomphateurs, en soulignant que Rome reste démocratique en parole. Sous cet angle, l'évergétisme vise moins l'expression d'une distance sociale que la transformation d'une magistrature déléguée en principe, en propriété ou droit subjectif. En ce qui concerne le pain, il est distribué par l'Etat. Les élections, elles, se font par réseaux et clientèles. L'évergésie n'est pas une monnaie d'échange en vue de l'obtention du pouvoir : les sénateurs sont évergètes parce qu'ils ont le pouvoir et parce que toute autorité cherche à se justifier. On retrouve l'idée d'une ambivalence irréductible du don civique qui se révèle ici «montrer au peuple qu'on se sent des devoirs envers lui et lui rendre hommage, montrer au peuple qu'on ne lui doit rien et qu'on possède une supériorité d'essence, à la manière d'un grand seigneur qui distribue des pourboires aux petites gens » (1976: 475). Si les notables municipaux donnent des jeux pour cultiver la distance sociale et parce que leur relation à la «grande politique » est problématique, les sénateurs, eux, le font pour manifester au peuple qu'il est leur obligé plus que leur mandant.

L'évergétisme impérial relève aussi d'une manifestation de la splendeur et de relations de dépendances mais il obéit à sa propre logique. Son style est plus monarchique que civique en ce qu'il exalte les vertus personnelles du souverain, le fait que l'obéissance relève moins du dévouement à la collectivité que de la confiance dans la providence impériale. Veyne résume le cœur de sa logique en soulignant qu'elle consiste à « présenter la loi impersonnelle comme une volonté individuelle du souverain vertueux, qui donne le pain et le cirque par évergétisme ; inversement, présenter l'individualité du monarque, qui se laisse voir au cirque, comme une incarnation de la majesté de l'Etat» (1976 : 478). Veyne rencontre ici la théorie des «deux corps du roi » et présente la «double personnalité » de l'Empereur (premier magistrat et évergète) comme reflet d'une bipolarité fondamentale de l'obéissance politique oscillant entre autonomie et hétéronomie: hétéronomie liée au fait d'obéir à autrui et autonomie liée au fait que je prétends toujours en même temps obéir pour mon bien. Soit l'autorité relève d'une délégation, soit ce n'est pas le cas et il faut que le roi règne parce qu'il est le maître, faisant dès lors le bien de ses sujets non parce qu'il est à leur service mais par évergétisme. Il faudra aussi invoquer Dieu pour justifier son droit de commander: l'évergétisme impérial va avec la divinisation des empereurs. Il est « une sorte de culte de l'universalité en l'individualité royale; il comprend deux espèces opposées, le style monarchique et l'apparat. Le premier rapporte l'universalité aux vertus individuelles du roi et le second confère à cette individualité une valeur universelle » (1976: 481). Le style monarchique impute à la bonté personnelle de l'Empereur les institutions publiques et les services administratifs qui organisent la vie quotidienne. Et réciproquement la personnalité royale revêt un caractère public ce qui implique le déploiement d'un apparat et la constitution de Rome en cour impériale. L'évergétisme est ainsi appréhendé sous l'angle de l'idéologie. «Sociologiquement, l'absence d'opinion entraîne politiquement la souveraineté par droit subjectif, laquelle entraîne idéologiquement l'évergétisme du souverain » (1976 : 492). Sous tout régime j'obéis à un autre pour mon bien, suivant des modalités de rapports plus ou moins impersonnelles. Dans le cas de l'Empire romain, l'idéologie rend compte de l'autonomie en mettant en relief la façon dont j'obéis pour mon bien, dont l'empereur règne par lui-même mais en même temps pour moi. Un souverain obéissant à un mandat n'a pas à faire des évergésies : on en attend de la sécurité, de la sincérité et de la simplicité. Mais d'un souverain comme l'Empereur, on attend à la fois la sécurité et la bonté : ainsi l'évergétisme recouvre le 
fait de tenir «sur le plan verbal » ses actes publics pour des dons. Il consiste aussi à tenir la personne privée de l'Empereur comme publique ce qui implique pour ce dernier d'exprimer sa majesté par des actes : «puisque tout régime est à la fois hétéronome et autonome, un souverain régnera par le droit qu'il en a, mais pour mon bien. On érigera alors son droit subjectif en droit divin ou en divinisation de l'Empereur et on érigera le fait qu'il règne pour mon bien en évergétisme impérial. Et puisque l'Etat, c'est lui, il déploiera l'apparat d'un gros propriétaire et l'Empire sera réputé être son patrimoine » (1976: 500). La notion d'idéologie renvoie précisément à cette idée d'un « apparat expressif ». Il ne faut pas la confondre avec la notion plus manipulatoire de propagande. En résumé, le roi, régnant par lui, est tenu de montrer qu'il ne règne pas que pour lui mais aussi pour les autres. Il a aussi besoin d'exprimer sa majesté : il ne s'agit pas de propagande mais de proclamer sa gloire y compris pour la postérité et, à la limite, souligne Veyne, même si personne n'écoute. La colonne Trajan exemplifie cette idée d'une majesté royale qui rayonne pour elle-même. En tant qu'il désigne des actes publics comme des dons, l'évergétisme a une portée dans tout l'Empire ; en tant que les pratiques de don relèvent de l'apparat, son périmètre est plus circonscrit à Rome comme Cour au sein de laquelle l'Empereur a le monopole de l'évergétisme.

\section{Un regard critique sur l'anthropologie du don.}

Telles sont résumées à grand trait les formes successives que prend l'évergétisme dans $L e$ Pain et le cirque. Dans cette section puis dans les deux suivantes, je m'attache à dégager les différents enjeux de ces analyses afin de souligner la façon dont elles apportent un éclairage sur les modes d'articulation possible entre don, reconnaissance et domination. Je reviens tout d'abord, en prenant notamment appui sur le travail d'Ilana Silber (2004), sur la façon dont la réflexion de Veyne procède d'un regard distancié et critique sur l'anthropologie du don.

Chez Mauss comme chez Veyne, on trouve une attention aux ambivalences et à la pluralité des logiques du don. Cependant, si Veyne se fait dans Le pain et le cirque à la fois historien et anthropologue, le regard reste prioritairement centré sur les singularités historiques, éloignant Veyne de toute réflexion sur le noyau universel (et potentiellement trans-historique) des pratiques de don. Or le travail de Mauss est en partie structuré autour d'une telle réflexion. Mauss ne néglige pas, loin de là, les variations historiques du don mais il entend tout de même en dégager des aspects voire une structure universelle et relativement constante. A l'inverse, Veyne ne cesse de souligner la pluralité des régimes possibles de don qu'il évite par ailleurs, à l'inverse de Mauss, de subsumer sous le terme d'échange. Là où Mauss met l'accent sur l'obligation de rendre comme un aspect invariant du phénomène du don, Veyne la relativise dans son analyse de l'évergétisme lorsqu'il souligne qu'il y a des dons appelés échanges ou des prestations déguisées en don mais aussi des formes de don qui se situent moins du côté de l'échange que du côté de l'entretien d'une relation d'amitié, de déférence, de protection : c'est le cas, par exemple, de l'évergétisme qui relève soit du cadeau, soit de l'hommage, c'est-à-dire de l'entretien d'une relation allant de haut en bas (de l'Empereur vers son peuple, des oligarques romains vers la plèbe) ou de bas en haut (si on envisage l'évergésie comme hommage à la cité). Le don est moins fondamentalement échange qu'épisode dans des relations personnelles ${ }^{1}$, il est lié à une dimension symbolique proprement anthropologique liée au fait que «tous les hommes sont sensibles aux signes extérieurs de mépris ou de respect, quels qu'ils soient, tous se contentent de commencements symboliques de preuves, faute de mieux » (1976: 88). Rien dans cette formule que Mauss n'accepterait d'endosser si ce n'est qu'aussitôt après Veyne ajoute que le don comme symbole est avant tout une convention qui

\footnotetext{
${ }^{1}$ Veyne semble parfois viser une version «échangiste » de l'anthropologie du don. Mais les travaux de C. Tarot (1999) ont plus récemment initié une réévaluation de la dimension symbolique et relationnelle du don maussien d'une façon qui pourrait converger avec le propos de Veyne.
} 
doit être agréée par la société. Sous cet angle, le discours de Veyne est à distance de celui, plus universalisant, de Mauss sur le don comme «roc des sociétés ». Comme l'a montré Silber, le volet d'histoire comparée de l'Essai sur le don met l'accent sur le dégagement des continuités entre pratiques de don à des époques différentes, sur la grammaire universelle qui peut les réunir. C'est tout l'inverse chez Veyne ne serait-ce qu'au seul niveau de l'analyse de l'évergétisme dont il souligne les variations en fonction, entre autres, des facteurs d'échelles (du passage du face à face civique à la configuration plus large de la "grande politique »). Un tel regard dérive d'ailleurs de la conception, défendue par Veyne (1979), du travail historique comme axé sur la mise en exergue des différences et la déconstruction des fausses continuités - conception bien démarquée de celle de l'Ecole des Annales et dont les lignes de convergence avec la problématique foucaldienne sont nombreuses. Il induit un souci non seulement de montrer que l'évergétisme renvoie à plusieurs systèmes ou formes de don civique mais aussi d'éviter tout rabattement de l'évergétisme sur d'autres phénomènes (potlatch, clientélisme, charité, impôt...). Dans le même ordre d'idée, Veyne va plus loin que Mauss dans la mise en exergue de la diversité et pluralité des mobiles pouvant présider aux pratiques de don (et parfois être combinés pour un même type d'acteurs et un même type de pratiques), du paternalisme à la peur de la guérilla sociale en passant par le goût de rivaliser... Cette priorité accordée aux différences et singularités a été critiquée car elle amènerait Veyne à sous-estimer les rapprochements potentiellement éclairants entre l'évergétisme et d'autres phénomènes (certaines formes de don religieux, la philanthropie moderne aux Etats-Unis). Il faut cependant signaler qu'elle est tempérée chez Veyne par le souci de débusquer des « invariants » mais là encore tout en s'écartant de l'anthropologie maussienne du don, à tout le moins de sa déclinaison structuraliste. Veyne dessine une sorte de voie moyenne entre Mauss et Levi-Strauss (1950) en soulignant que les règles du don ne sont pas conscientes de sorte qu'il ne faut pas croire les Romains sur parole (comme on a reproché à Mauss de l'avoir fait en ce qui concerne le discours de Tamati Raipiri sur le hau) mais elles ne sont pas inconscientes non plus : elles relèvent d'une grammaire pré-conceptuelle. Dégager des invariants ne revient donc pas à faire émerger des structures mentales inconscientes mais à dégager des «thèmes » récurrents qui n'existent pas «à l'état sauvage » et sont soumis à des modifications constantes. Dans le cas de l'évergétisme, les thèmes mis en avant sont l'évergétisme comme tendance à exprimer et déployer ses possibles, l'évergétisme comme obligation (liée à la dignité de charges) et la préoccupation pour l'au-delà mais on comprend qu'il s'agit là d'invariants «toujours provisoires, jamais systématiques, jamais détachés des cas concrets à partir desquels ils ont été élaborés » (1976:47).

Enfin, il faut ajouter d'autres démarcations avec l'anthropologie du don aussi bien avec celle élaborée par Mauss qu'avec celle dont le travail de Karl Polanyi (1944) dessine les contours. Tout d'abord Veyne éclaire les dimensions plus politiques du don se rapportant aux rapports d'autorité : en cela, on y revient dans la dernière section, son travail constitue une passerelle entre l'optique du don et la réflexion wébérienne sur les formes de domination. Ensuite, Veyne met à distance - et ce n'est pas sans lien probablement avec l'influence wébérienne les implications politiques (voire normatives) de l'anthropologie du don qui, chez Polanyi comme chez Mauss, s'articule à la défense d'un socialisme démocratique. Le propos wébérien de Veyne sur l'inévitable scission gouvernants-gouvernés induit un regard sceptique sur ce type de discours. Enfin, Veyne critique directement les thèses de Polanyi suivant lesquelles les économies pré-industrielles n'étaient pas organisées autour d'une centralité du marché mais suivant des formes de réciprocité et de redistribution. Polanyi a en effet mis en relief trois grands modèles institutionnalisés d'articulation des éléments du processus économique : la réciprocité qui implique une symétrie, la redistribution impliquant des mouvements d'appropriation vers un centre puis de ce dernier vers l'extérieur, l'échange qui implique des mouvements de va-et-vient (l'intégration étant dans ce cas conditionnée par un système de 
marché créateur de prix). Les systèmes sociaux ayant existé jusqu'à la fin de la féodalité en Europe occidentale ont été organisés suivant les principes de la réciprocité ou de la redistribution, ou encore de l'administration domestique (production et stockage de ressources pour un groupe fermé) voire d'une combinaison des trois alors que le système capitaliste se caractérise par la domination de l'échange. Si c'est surtout sous le vocable de réciprocité que Polanyi subsume les pratiques de don décrites par Malinowski au travers du commerce kula (caractérisé par une symétrie des villages), il est effectivement tentant de subsumer une pratique comme celle de l'évergétisme, impliquant un centre (élite, Empereur) sous la redistribution. Mais Veyne résiste à une telle lecture, reprochant à la redistribution de mélanger trop de choses, des trocs intéressés, des offrandes symboliques et des «déguisements idéologiques »: «la redistribution prend en écharpe le troc qui a une fin économique et le legs que fait un mourant à l'Eglise pour le salut de son âme et qui a seulement un matériau économique; la réciprocité et le marché semblent s'exclure comme deux systèmes alternatifs, alors qu'en réalité la réciprocité est souvent un marché à l'état naissant et que, quand elle ne l'est pas, quand elle est échange symbolique de bons procédés et d'invitations à dîner, elle n'est pas propre à servir de solution alternative au marché » (1976: 80).

\section{Critique du fonctionnalisme et du schème de la dépolitisation}

Dans la lignée de cette critique du motif de la redistribution, Veyne refuse, tout autant que l'identification de l'évergétisme à la charité (il admet bien une réorientation progressive des dons du domaine civil vers le domaine religieux vers le IVème siècle mais soutient que la logique de l'évergétisme reste prioritairement civique et patriotique ${ }^{2}$ ), son rabattement sur un impôt ne serait-ce que parce que les évergésies sont souvent plus conformes aux vœux du mécène qu'à ceux de la collectivité, les évergètes voulant d'abord se faire plaisir à euxmêmes et ayant d'autres motifs de donner qu'un contribuable. Il arrive qu'ils répondent à une forme de pression sociale en donnant du blé, de la viande sans que évergétisme et fiscalité soient articulés l'un à l'autre : l'évergétisme ne remplace pas des revenus insuffisants - au contraire il est lié à la prospérité de la cité - et il ne se proportionne pas aux revenus et besoins de la collectivité. Là où le système fiscal implique qu'un grand nombre verse un peu, l'évergétisme recouvre le fait qu'un petit nombre donne beaucoup ; là où la redistribution fonctionne en système, l'évergétisme correspond à un jeu " au coup par coup ». Derrière ces analyses, il faut voir à l'œuvre tout autant qu'une critique de Polanyi, le refus d'identifier l'évergétisme à une fonction facteur d'équilibre du système social. Si l'évergétisme peut être identifié à une fonction ce n'est jamais que de façon secondaire. Il n'est pas étonnant que Jon Elster (1986 et 1990) qui a consacré une partie de ses travaux à une critique du fonctionnalisme en sciences sociales, se réclame à cet égard de Veyne (même s'il lui reproche parfois d'avoir lui-même versé dans cet écueil par exemple en caractérisant l'évergétisme

\footnotetext{
${ }^{2}$ Sur ce point, les travaux de P. Brown (1993) complètent ceux de P. Veyne en revenant sur la christianisation de l'Empire Romain et ses effets sur l'évolution des modalités de l'exercice de l'autorité (du fait de la montée en puissance des évêques et du «culte des saints »). Ils éclairent la transformation des schémas de don public qui ne se structurent plus autour du don à la cité mais des pratiques religieuses de don visant les pauvres. Cette transformation participe d'une évolution du modèle de société qui structure les façons de penser. Et, dans la lignée de Veyne, Brown montre comment ces nouvelles pratiques de don public sont une manière pour de nouvelles élites de se structurer et de s'exprimer (en découlent des formes de chevauchement et de rivalité entre évergétisme et don religieux), il dégage les logiques de reconnaissance entre élite religieuse et bénéficiaires des dons mais aussi au sein de l'élite elle-même. Les pratiques de don contribuent à structurer et faire reconnaître une identité collective-statutaire. C'est pourquoi Silber (2013) souligne les dynamiques symboliques de reconnaissance qui traversent la charité chrétienne aussi bien que l'évergétisme et la façon dont tous deux apparaissent, au sein des travaux de Brown et Veyne, comme autant de ressorts de «gouvernementalité » au sens foucaldien du terme. On peut cependant ajouter ici que, à la différence de l'analyse de Veyne, celle de Brown assume une tonalité plus fonctionnaliste (Brown s'inscrit d'ailleurs dans la tradition durkheimienne).
} 
comme une «barrière » d'argent filtrant l'accès au politique) et de son regard sceptique sur toute conception trop rationaliste et «bien huilée » des formes sociales. Elster a fait son miel de l'insistance de Veyne sur le fait que les évergètes ne cherchent pas l'admiration du peuple : ils cherchent à exprimer la distance sociale et la popularité n'est qu'un effet secondaire. Elster en a tiré un précieux éclairage sur ces objectifs qui ne peuvent être que des effets secondaires d'autres actions (comme la spontanéité, l'oubli ou l'obtention d'estime qui ne peut faire l'objet d'une demande directe). De même, le travail d'Elster sur le phénomène d'adaptation des préférences aux contextes de choix, prolonge l'insistance de Veyne sur la catégorisation de l'équilibre et solidairement du pacte social comme fiction normative participant de processus de réduction des «dissonances cognitives»: «les hommes se passent de l'équilibre : ils ne connaissent que leur horizon et tentent de s'y adapter » (1976:318) sans faire toute une question de l'équilibre et de l'égalité. L'évergétisme ne peut d'ailleurs jouer le rôle de «ciment » de l'ordre social, en apaisant l'envie, car il suscite autant de ressentiment que de reconnaissance (comme gratitude). Et Veyne rejette toute explication de l'évergétisme par le fait qu'il satisfait les intérêts des dominants au nom de la tendance qu'ont les intérêts à s'accommoder des rapports de domination et de la façon dont l'autorité est susceptible de reposer en partie sur des mécanismes de réduction de la dissonance cognitive : «la relative stabilité des sociétés, entre l'idylle et le chaos, ne tient pas à un juste équilibre qu'elles auraient réalisé entre leurs membres, mais au fait que l'humanité ne se pose de problèmes que lorsqu'elle peut les résoudre » (1976: 327). Le pain et le cirque ne s'expliquent pas par la façon dont ils assurent la paix sociale même si l'on peut éventuellement envisager qu'ils contribuent à ne pas la rompre.

La critique du fonctionnalisme a partie liée ici avec une critique du marxisme qui se fait jour au fil des pages. Certes le marxisme que Veyne cible a probablement une dimension durcie voire, oserait-on dire, caricaturale mais il n'en demeure pas moins que Veyne en atteint l'un des noyaux en pointant les limites de la notion d'intérêt de classe. Il récuse en effet l'idée que l'évergétisme s'explique par la sauvegarde de rapports de production. On retrouve la récusation d'un fonctionnalisme rampant mais aussi l'idée que les intérêts des notables comme évergètes ne sont pas nécessairement ceux des notables comme possédants : «les hommes ne cherchent pas par essence à défendre les rapports de production, mais bien leur distance sociale, s'ils en ont une et quoi que ce soit qui leur confère cette distance ; les notables n'étaient pas magistrats et évergètes pour défendre leurs propriétés foncières, mais parce que la notabilité les séparaient du peuple » (1976: 332). Il ne s'agit pas de conserver des rapports de production même si la distance sociale passe par la richesse (mais celle-ci conditionne la distance sans s'y identifier). On serait tentés, même si Veyne, ne le formule pas ainsi de souligner une possible influence de l'approche wébérienne de la stratification et de la façon dont elle refuse de faire du statut une simple idéologie, un mode de légitimation des rapports économiques (Weber maintenant une autonomie relative du statut comme honneur social par rapport à la classe et insistant sur la pluralité des facteurs - économiques, culturelles et politiques - de stratification). Enfin, quand il envisage tout de même l'évergétisme comme matière d'une idéologie, Veyne en récuse certaines conceptions qu'il associe au marxisme, celle de l'idéologie comme reflet ou idée planant au-dessus des consciences mais surtout comme conduite rationnelle correspondant à des calculs. Veyne reprend ici l'exemple, emprunté à La Fontaine, du renard et des raisins ${ }^{3}$ - dont Elster s'est inspiré lui aussi - pour souligner que l'idéologie est liée au moins autant au besoin qu'ont les dominés de justifier leur situation de relative impuissance qu'au souci des dominants de légitimer leur domination. L'évergétisme comme croyance en la bonté d'un Empereur ne sert pas à rendre les sujets dociles : c'est plutôt leur docilité qui produit la croyance. En ce sens,

\footnotetext{
${ }^{3}$ De ce renard qui, de façon fameuse, ne pouvant atteindre de magnifiques raisins couverts d'une peau « vermeille», en conclut qu'ils sont « trop verts » et «faits pour les goujats ».
} 
dit Veyne, l'évergétisme comme idéologie ne reproduit pas l'ordre établi mais c'est sa reproduction qui induit le maintien de cette croyance dans l'esprit des sujets. En outre, il met l'accent (on l'a déjà mentionné) sur la dimension expressive de l'idéologie de l'évergétisme comme apparat afin d'évacuer toute lecture de celle-ci en termes de mystification et de propagande. En cela encore, Veyne se démarque d'un certain discours marxiste.

C'est ainsi que les analyses de Veyne nourrissent un regard sceptique sur toute analyse du don en termes de dépolitisation. Le schéma de la dépolitisation a pris des formes diverses se traduisant soit par des discours moralisant sur la corruption du peuple par les privilégiés, soit par la dénonciation typiquement radicale ou marxiste des stratégies de diversion des dominants (détournant les masses des luttes contre les inégalités). On ne rendra jamais assez hommage au caractère incisif des analyses de Veyne à cet égard. Elles mettent en lumière le caractère normatif du schéma de la dépolitisation et ses incohérences: "L'idée de dépolitisation va de contradiction en contradiction, elle commence par idéaliser les gens : l'autonomie politique serait inscrite dans leur essence ; puis elle les met plus bas que terre : il leur suffit de leur proposer le cirque pour les dénaturer : elle les relève en imputant leur aliénation à la baguette magique du tyran. Pour un rêve d'autonomie politique, elle dénie leur autonomie anthropologique » (1976: 98). Il faut donc considérer non pas que l'évergétisme vise à dépolitiser le peuple en produisant une dissymétrie gouvernants-gouvernés qui n'aurait pas existé sans lui mais plutôt que les élites oligarchiques et l'Empereur auraient sans doute politisé le peuple s'ils ne lui avaient pas donné le pain et le cirque.

\section{Don, prestige et domination : Veyne entre Veblen et Weber.}

Ce type d'analyse nous reconduit à nouveau au caractère expressif des rapports entre don et pouvoir, à la notion problématique d' «ostentation». Comme Mauss dans son analyse des pratiques de don dans les îles Trobriand, Veyne mobilise la conception aristotélicienne de la magnificence comme art de donner sans recevoir en échange ${ }^{4}$, qu'il juge utile pour rendre compte de l'évergétisme. Si cette magnificence est à la fois libre et forcée, ce n'est pas parce qu'elle répondrait à une pression de l'opinion (même si elle peut le faire) mais parce qu'elle recouvre la tendance que nous avons, dès que nous pouvons nous prévaloir d'une forme de supériorité ou d'excellence à la déployer. On retrouve ici l'un des trois invariants mis en avant par Veyne pour rendre compte de l'évergétisme. Veyne le résume parfois par la notion d'ostentation mais il prend aussi ses distances avec cette notion forgée par Thorstein Veblen. Dans cette section conclusive, je montre comment l'analyse par Veyne du caractère expressif du rapport entre évergétisme et domination se situe en quelque sorte entre Veblen et Weber. Puis j'insiste sur la façon dont c'est l'insistance sur cette dimension expressive qui constitue le fil directeur à partir duquel Veyne établit une passerelle entre Mauss et Weber.

Il est de fait tentant de rendre compte de l'évergétisme à partir d'une notion comme celle, élaborée par Veblen, de «consommation ostentatoire». Veblen a forgé cette notion dans la Théorie de la classe de loisir sur la base d'une anthropologie évolutionniste : c'est, entre autres, à partir d'un socle d'instincts - dont la plasticité est soulignée - que Veblen analyse l'évolution des formes sociales, en insistant sur l'antagonisme entre un instinct artisan (du travail bien fait) et un instinct prédateur lui-même fortement articulé à un instinct de rivalité ou de «comparaison envieuse ». Ce sont ces deux instincts qui s'expriment dans les pratiques ostentatoires de consommation des riches américains de la classe oisive. La notion même de « consommation ostentatoire » renvoie au choix de modes de vie ou de biens pouvant jouer le rôle de marqueurs statutaires car ils recouvrent des formes de gaspillage et peuvent signifier, pour autrui, la capacité à s'exempter du travail « utile»: elle fait fond sur une dévalorisation du travail et une valorisation du loisir comme critère de prestige. Le fameux « effet Veblen »

\footnotetext{
${ }^{4}$ Voir le livre IV de l'Ethique à Nicomaque.
} 
renvoie au paradoxe qui fait que plus certains objets sont chers, plus leur consommation augmente alors même qu'ils ne correspondent à aucun des besoins " génériques » de la vie. La consommation « ostentatoire » consiste dans le fait de choisir des objets (voiture, maison, vêtements) pour des critères qui n'ont rien à voir avec la fonctionnalité mais tout avec l'effet produit aux yeux d'autrui. L'instinct prédateur conduit à traiter objets et individus (femmes, domestiques, animaux en particulier) comme autant de trophées qui signifient le statut de propriétaire et la capacité à se soustraire au travail de subsistance. Les vêtements mêmes (notamment féminins) sont d'autant plus valorisés qu'ils entravent toute forme de travail. La notion de consommation ostentatoire est articulée à une analyse du système capitaliste comme caractérisé par le gaspillage voire par le sabotage de l'activité industrielle (afin de maintenir des prix élevés).

L'évergétisme ne relève pas d'une forme de consommation mais pourrait être appréhendé en termes de don ostentatoire ou « de prestige ». Veyne souligne néanmoins que son analyse en termes de distance sociale constitue une avancée par rapport à celle de Veblen. Il reproche à la notion d' "ostentation » sa tonalité morale : cette notion occulte le fait que le don civique articule contrainte et liberté. Même si l'évergétisme est moins « contraint » que les liturgies, il ne relève pas d'actes «libres » ou non-contraints. La contrainte est plurielle : elle est liée à la nécessité pour toute supériorité sociale de s'exprimer mais elle est aussi collective, liée à la pression interne au groupe des notables. L'analyse de Veyne converge avec la critique de Veblen par Norbert Elias dans La société de cour. Analysant les pratiques de dépenses somptuaires de la noblesse, Elias reproche à Veblen de s'être enfermé dans un discours de satire sans avoir perçu que les dépenses de prestiges des couches supérieures relèvent d'une nécessité à laquelle elles ne peuvent se soustraire sous peine de mort sociale : Veblen n'aurait pas intégré la riche intuition de Max Weber sur la façon dont le luxe relève dans ce cadre non du superflu mais de la nécessité absolue d'affirmer son rang. Veyne et Elias se rejoignent dans l'accent mis sur le continuum entre contrainte et liberté ou spontanéité : ces termes sont les facettes d'une même médaille au point que Veyne souligne que la spontanéité est démultipliée par la contrainte. La notion d'ostentation suggère celle de vanité et celle de narcissisme. Elle a l'inconvénient de mettre en avant un travers individuel. C'est pourquoi Veyne lui préfère celle d'apparat comme institution visant autrui : «Toute supériorité qui ne se fait pas voir devient suspecte : ne serait-elle pas usurpée ? La consommation ostentatoire sert moins à s'attirer l'estime, comme dit Veblen, qu'à ne pas la perdre : il faut «tenir »son rang. L'apparat n'ajoute pas grand chose aux sentiments du spectateur, qui n'en attendait pas moins, mais son absence la décevrait. L'apparat est une sorte d'institution, en ce sens qu'il répond à une attente générale » $(1976: 111)$. Il articule donc spontanéité et contrainte et comporte une dimension collective car il constitue un devoir de classe pour les couches supérieures. Veyne met en outre à distance le puritanisme de Veblen (le reproche est classique) en soulignant par ailleurs que l'idée d'un narcissisme ostentatoire ne rend pas compte du phénomène d'acculturation à la richesse ainsi que du fait que la richesse est une excellence généralement reconnue sur le plan social : la tonalité satirique de l'analyse de Veblen ne permettrait pas de dégager la logique institutionnelle à l'œuvre derrière les pratiques de dépense ou de don de prestige. C'est pourquoi j'ai souligné que l'analyse de Veyne se situe entre Veblen et Weber (ou Elias qui se réclame de ce dernier dans son analyse du luxe). Veyne prend ses distances avec Veblen au nom d'une sensibilité plus grande à la façon dont les évergètes évoluent au sein de configurations comportant des formes de contraintes importantes. Cependant il reste permis de penser que Veyne a une certaine « dette » à l'égard de Veblen. Si le discours de Veblen prend parfois l'allure d'un discours de satire non exempt d'une tonalité morale, il ne méconnaît pas non plus totalement la part de contrainte inhérente aux logiques de prestige. Et lorsque Veyne insiste sur la tendance de toute supériorité ou distinction à se déployer, il reprend là une intuition forte de Veblen. 
C'est l'insistance sur cette dimension expressive plutôt qu'instrumentale des rapports entre don et domination, qui constitue le fil conducteur à partir duquel Veyne établit une passerelle entre Mauss et Weber. On l'a déjà mentionné, Veyne reprend et prolonge des intuitions wébériennes sur la nécessité pour toute domination de se légitimer, sur l'irréductible dissymétrie, dans toute forme sociale, entre gouvernants et gouvernés. Dans le même temps, il entreprend de corriger ou d'affiner les analyses wébériennes sur certains points : en traitant le puritanisme comme une aggravation accidentelle de l'esprit du capitalisme plus que comme un de ses éléments structurants (le capitalisme étant lié selon Veyne à une tendance anthropologique de l'homme à actualiser ses capacités rationnelles); ou en allant au-delà de la définition vague du charisme comme qualité extraordinaire d'un individu. Veyne distingue différentes formes de charisme : le charisme royal correspondant le plus à ce que Weber envisage comme le charisme lié à la fonction, le charisme héroïque qu'un individu conquiert par ses prouesses et le charisme de leadership qui ne se développe que dans des sociétés de masse où prime la logique de délégation de l'autorité. Par contraste avec le charisme royal résultant de l'hétéronomie, le charisme de leadership se développe dans un contexte de crise et correspond à un retournement de l'autonomie collective contre elle-même, la société s'adorant elle-même et se rassemblant d'autant plus dans l'exaltation du chef qu'elle entreprend de «mauvais coups risqués ». A travers la figure de Staline, Veyne introduit aussi la figure intermédiaire du charisme de "génialité » qui correspond à la tentative désespérée de restaurer une autorité de droit subjectif à une époque où seule la logique de délégation est légitime : le charisme du «génie » recouvre un pastiche du charisme de prouesse et va de pair avec une existence de «fonctionnaire en uniforme»(1976: 522) là où les Empereurs romains, passant pour les propriétaires de l'Empire, étaient à la fois les « maîtres de céans » et de bons maîtres, affichant cette libéralité naturelle qui les rendait évergètes. L'évergétisme apparaît donc lié avant tout au charisme royal, il n'est pas l'un des moyens de sa production mais plutôt l'un de ses modes d'expression possibles. Sous cet angle, on peut souligner que Veyne enrichit la perspective de Weber qui ne s'est jamais beaucoup préoccupé des pratiques de don si ce n'est sous un point de vue trop étroitement instrumental et utilitaire (Silber, 2010). Réciproquement il enrichit l'anthropologie du don en établissant un pont avec la sociologie wébérienne de la domination en faisant ressortir les logiques politiques du don comme modalité de l'autorité .

Et c'est sur cette base comme le souligne Silber, que le travail de Veyne a eu le mérite d'être l'un des premiers à attirer l'attention sur la capacité des pratiques de don à symboliser des identités aussi bien collectives qu'individuelles dans l'espace public. Le Pain et le cirque constitue donc un texte d'une richesse exceptionnelle dès lors que l'on entreprend de réfléchir aux rapports entre don, domination et reconnaissance. Il établit un pont entre Weber et Mauss en attirant notre attention sur les logiques politiques du don et la façon dont elles nous disent quelque chose des rapports d'autorité. Il attire notre attention de façon subtile sur des mécanismes sociaux qu'il est nécessaire d'intégrer pour comprendre les logiques de production de l'estime sociale : il nous met en garde contre toute tentative de chercher une équation simple pour penser les rapports entre le don et la reconnaissance comme prestige ; en effet il éclaire à la fois la façon dont les pratiques du don civique sont liées à un désir certain de reconnaissance comme prestige mais aussi la façon dont elles ne peuvent susciter de l'admiration du peuple et de la popularité qu'à condition de ne pas la viser directement. En cela, Veyne souligne l'irréductible ambivalence des rapports entre don et reconnaissance dans l'évergétisme qui peut consister à la fois à rendre hommage à la collectivité et à lui montrer qu'on ne lui doit rien. L'attention fine à ces ambivalences et la critique de la vision rationaliste, instrumentale des rapports don-domination constituent des apports précieux du

\footnotetext{
${ }^{5}$ Veyne a tendance, comme Weber, à identifier domination et autorité (comme impliquant la légitimation de la puissance).
} 
travail de Veyne qui nous met en garde contre la tentation de catégoriser trop rapidement le don comme un vecteur de production de la domination et de l'équilibre social : le don est sans doute souvent plus une modalité d'expression de rapports de pouvoir que «moyen » de les produire.

\section{Bibliographie}

Bourdieu P. (1972/réédition 2000), Esquisse d'une théorie de la pratique, précédée de Trois études d'ethnologie kabyle, Paris, Seuil.

Bourdieu P. (1997), Méditations pascaliennes, Paris, Seuil.

Brown P. (1993), Pouvoir et persuasion dans l'Antiquité tardive, Paris, Seuil.

Elias N. (1985), La société de cour, Paris, Flammarion.

Elster J. (1986), Le laboureur et ses enfants, Paris, Minuit.

Elster J. (1990), Psychologie politique, Paris, Minuit.

Hénaff M. (1990), Le prix de la vérité, Paris, Seuil.

Hénaff M. (2012), Le don des philosophes. Repenser la réciprocité, Paris, Seuil.

Lefort C. (1951), «L'échange et la lutte des hommes », Les Temps Modernes, t. 6, LXIV, p. 1400-1417.

Levi-Strauss C. (1950/2012), Introduction à l'œuvre de M. Mauss, Paris, PUF.

Malinowski B. (1963), Les Argonautes du Pacifique occidental, Paris, Gallimard.

Polanyi K. (1944/1983), La grande transformation, Paris, Gallimard.

Silber I. (2004), «Entre M. Mauss et P. Veyne : pour une sociologie historique comparée du don », Sociologie et sociétés, vol. 36, numéro 2, 2004, p. 189-205.

Silber I. (2010), «Mauss, Weber et les trajectoires historiques du don », Revue du Mauss, 2010/2, numéro 36, p. 539-61.

Silber I. (2013), « Neither Mauss nor Veyne : Peter Brown interpretative path to the Gift », Satlow M. éd., The Gift in Antiquity, John Wiley \& Sons, p. 203-220.

Mauss M. (1925/2012), Essai sur le don, Paris, PUF.

Tarot C. (1999), De Durkheim à Mauss, l'invention du symbolique. Sociologie et science des religions, Paris, La Découverte.

Veyne P. (1976/1995), Le pain et le cirque. Sociologie historique d'un pluralisme politique, Paris, Points Seuil.

Veyne P. (1979), Comment on écrit l'histoire, Paris, Seuil.

Veblen T. (1979), Théorie de la classe de loisir, Paris, Gallimard.

Weber M. (2014), La domination, Paris, La Découverte. 